\title{
Effect of deprivation level on responses to novel alleys in albino rats'
}

\author{
JEROME S. COHEN AND LAURENCE J. STETTNER, \\ DEPARTMENT OF PSYCHOLOGY, WAYNE STATE \\ UNIVERSITY, Detroit, Mich. 48202
}

After learning to run an L-shaped alley for sucrose solution, male albino rats were presented novel alleys $(N$-alleys $)$ in the same and opposite direction as the goal turn-off alley. Moderately water deprived Ss (MOD group) entered both types of N-alleys more frequently than highly deprived Ss (HI group). MODs also showed the typical exploration gradient, of entering alleys nearest the start box more frequently than those nearer to the goal. HIs, however, entered an $\mathrm{N}$-alley on the goal side most frequently when it was nearest the goal. The results were interpreted as based on combined goal anticipatory and exploratory behavior.

A recent study by Cohen \& Stettner (1968) found that exploratory activity decreases with high levels of drive. Exploration in that study consisted of entry into a novel blind alley (N-alley) attached to a runway. The rats had already learned to run straight in order to obtain sucrose solution reinforcement. Hughes (1964) and the present authors (1968) suggested that results of other studies that had found an increase in exploration with higher drive levels (Glickman \& Jensen, 1961; Stackhouse et al, 1960; Adlerstein \& Fehrer, 1955) might have included food and water seeking anticipatory goal activities along with exploratory tendencies.

The present study further examines this problem by systematically varying conditions to enhance or inhibit goal anticipation responses compared with goal competing exploration. By using an $\mathrm{L}$-shaped alley, we can make a turn into an $\mathrm{N}$-alley either similar to or opposite from the turn necessary to enter the goal area. On the goal side, Ss might enter the $\mathrm{N}$-alley in anticipation of reinforcement as well as for exploration. Clearly, higher drive levels should produce more anticipatory entries into the $\mathrm{N}$-alley or the goal side, so that more highly deprived (HI) rats should make more entries on this basis than moderately deprived (MOD) animals. Further, as discussed by Hull (1943) and shown by Deaux \& Patten (1964), anticipatory goal activity increases as an animal moves closer to the goal. Therefore, not only should HI Ss emit more anticipatory activity, but should enter an $\mathrm{N}$-alley on the goal side more frequently the closer it is to the goal end of the runway. This would disrupt the normal "exploration gradient" of $\mathrm{N}$-alley entry (Cohen \& Stettner, 1968); wherein frequency of $\mathrm{N}$-alley entry decreases the closer it is to the goal. Thus, the spatial pattern of results can serve to differentiate anticipatory and exploratory types of $\mathrm{N}$-alley entry. Furthermore, the completeness of the $\mathrm{N}$-alley entry as well as the actual number of responses should indicate the strength of the reaction to the $\mathrm{N}$-alley. For HI Ss, since goal anticipation is presumably very high, more WhB (whole body) entries should be made to the goal side than the opposite side. Similarly, WhB entries should account for the spatial gradient of goal anticipation. With MOD Ss, since goal anticipatory tendencies are low but exploratory tendencies high, these response types should display similar exploratory patterns on both sides.

Subjects. The Ss were 24 male Sprague-Dawley albino rats, 100 days old at the time of training. Half of the Ss were maintained on an average of $24 \mathrm{~h}$ of water ad lib within $48 \mathrm{~h}$ (MOD group). HI group Ss were deprived $46 \mathrm{~h}$ and allowed $2 \mathrm{~h}$ ad lib drinking per $48 \mathrm{~h}$.

Apparatus. The main training runway consisted of a straight alley $(92 \times 4 \times 5$ in.) with 15 removable wooden partitions lining both side walls. At the last pair of partitions, one was removed and another alley ( $36 \times 4 \times 5 \mathrm{in}$.) was placed perpendicular to the main stem, forming an L-shaped runway. The start chamber (13 in.) was situated at the beginning of the longer runway, and the goal chamber (13 in.) which contained a drinking tube set 15 in. above the floor, at the end of the turn off alley. Both chambers were set off by guillotine doors. A photoelectric beam was situated $1 \frac{1}{2}$ in. above the floor in front of the drinking tube. When the door of the start box was lifted, a standard clock was started. The intersection of the photoelectric beam by $S$ stopped the clock. In this way, $S$ 's running time was recorded for each trial. Novel exploratory alleys ( $\mathrm{N}$-alleys) of the same dimensions as the goal turn alley could be placed perpendicular to the straight runway at any of the positions when the appropriate wooden partition had been removed. All interiors were painted flat grey.

Training. The Ss received all trials at the end of their respective deprivation period. A training trial consisted of $S$ running down and negotiating the turn at the end of the runway and drinking. After a 10-sec drink of the $10 \%$ sucrose solution, $S$ was removed, handled for $20 \mathrm{sec}$, and placed in the start chamber again. All Ss received ten 10-trial blocks (one block every other day) or 100 trials. Half the $\mathrm{Ss}$ in each deprivation group learned this task with the goal turn alley on the left side and the other half with this alley on the right.

Novel-Alleys. After the training trials, two $\mathrm{N}$-alleys were introduced at any one of three locations: directly after the start door (position 1); at the middle of the straight runway (position 2); or 8 in. in front of the goal turn off alley (position 3) The Ss were not removed until they had entered the goal alley and obtained reinforcement, just as in training. Three 10-trial blocks of $\mathrm{N}$-alley presentation trials were run. $\mathrm{N}$-alleys appeared at any one distance at the same time so that $S$ could choose to enter either left or right at that point. For each block, the $\mathrm{N}$-alleys were placed in a different position. Each $\mathrm{S}$ was assigned to one of three $\mathrm{N}$-alley position schedules: $123,231,312$. "231," for example, means that on the first day of novel alley trials, both $\mathrm{N}$-alleys were in position 2, on the second day, they were in position 3 and on the third day in position 1 . The type of $\mathrm{N}$-alley-entry made by $\mathrm{S}$ was noted. A partial (HS) response consisted of only a 2 in. entry, while a complete (WhB) response consisted of a 5 in. entry by $S$.

Any $S$ who refused to enter the goal area and drink during any one training or post training trial within $5 \mathrm{~min}$ was removed from the study. This method of $\mathbf{S}$ selection was instituted after we had observed in pilot studies that $\mathrm{N}$-alley entry also constituted an attempt for escape by fearful Ss. This criterion led to the removal of $6 \mathrm{HI}$ and 15 MOD Ss before $12 \mathrm{Ss}$ in each group could be obtained. Removal of fearful or too lowly motivated Ss would tend to attenuate actual drive level differences among the groups, thus tending to bias results against finding differences between groups.

Results and Discussion. The basic effect of drive level manipulation was reflected in faster running speeds over the last three training blocks for HI Ss than for MOD Ss (mean latency $2.91 \mathrm{sec}$ or mean reciprocal speed .343 vs $3.94 \mathrm{sec}$ or $.254, \mathrm{t}=4.32$, $\mathrm{df}=$ $22, \mathrm{p}<.001)$.

The frequency of $\mathrm{N}$-alley entries is shown in Table 1. (Figures in parentheses show entry choices only for the very first $\mathrm{N}$-alley trial. As can be seen, trends for these data are the same as for the overall

Table 1.

Summary of Total Entry Frequency Data and Choice on the First N-Alley Trial

(in parentheses)

\begin{tabular}{|c|c|c|c|c|c|}
\hline \multicolumn{6}{|c|}{ N-Alley Location } \\
\hline \multicolumn{3}{|c|}{ Same Side } & \multicolumn{3}{|c|}{ Opposite Side } \\
\hline 1 & 2 & 3 & 1 & 2 & 3 \\
\hline
\end{tabular}

Entry type for HI group

$\begin{array}{llcclrc}\text { HS } & 10(5) & 8(6) & 4(2) & 21(17) & 4(3) & 8(4) \\ \text { WhB } & 31(25 & 12(8) & 48(41) & 19(8) & 11(4) & 18(8) \\ \text { Total } & 41(30) & 20(14) & 52(43) & 40(25) & 15(7) & 26(12)\end{array}$

Entry type for

MOD group

$\begin{array}{lllllll}\text { HS } & 32(21) & 12(7) & 19(8) & 39(15) & 21(14) & 24(10) \\ \text { WhB } & 51(16) & 34(21) & 27(22) & 42(21) & 32(16) & 32(20) \\ \text { Total } & 83(47) & 46(28) & 46(30) & 81(36) & 53(30) & 56(30)\end{array}$


entries, indicating that the totals were not appreciably confounded by effects within the blocks of $\mathrm{N}$-trials). An analysis of variance was carried out for all entries and for each type of entry. More entries were made by MOD Ss than by HI Ss $(F=19.87, \mathrm{df}=$ $1 / 22, p<.01)$. This higher MOD entry frequency was accounted for by both significantly more HS entries $(F=22.10, \mathrm{df}=1 / 22$, $p$ $<.01)$ and $\mathrm{WhB}$ entries $(\mathrm{F}=4.97, \mathrm{df}=1 / 22, \mathrm{p}<.05)$. Individual comparisons (Neuman-Keuls) revealed that MOD Ss entered the $\mathrm{N}$-alleys on each side significantly more $(\mathrm{p}<.01)$ than HI Ss. This was reflected for both entry types well above the .01 critical level.

The action of goal anticipation is readily seen in the HI group by the greater number of $\mathrm{N}$-alley entries made on the goal side ( $\mathrm{p}$ $<.01$ ), and by the predominance of position 3 in eliciting more goal side entries than position $2(\mathrm{p}<.01)$ or position 1 (trend not significant). Closer analysis reveals that $\mathrm{WhB}$ entries were solely responsible for these entry patterns. Indeed, on the opposite side more of the previously seen (Cohen \& Stettner, 1968) exploratory pattern prevailed, that is more entry activity at position 1 than 2 $(p<.05)$ or 3 (not significant). Although both entry types show this pattern, only HS entries were significantly greater $(p<.05)$ at position 1 than 2 . Indeed this exploratory trend was also slightly evident for HS entries along the goal side for this group.

For MOD Ss, on the other hand, there was no significant difference in entry activity between either side, although significantly more HS entries were made to the opposite side $(\mathrm{p}<.01)$. On each side, position 1 elicited more entries than the other two positions $(\mathrm{p}<.01$ for both comparisons), the typical exploration gradient. Both entry types exhibit this pattern $(p<.05$ for HS both sides and $p<.05$ for WhB same side, trend only on opposite side).

These results confirm the belief that entries into the $\mathrm{N}$-alleys for HI Ss stem from at least two different sources: exploratory tendencies and anticipatory tendencies. MOD Ss seem to be operating under predominantly stronger exploratory tendencies which were sufficient to overcome the greater goal anticipatory tendencies of HI Ss. Although WhB entries were responsible for the spatial gradient and entry frequency differences for HI Ss, we cannot equate WhB responses solely with anticipation. The increased number of these responses is best interpreted as reflecting anticipatory responses, however.
Such an interpretation, though, does not explain the fact that more WhB entries were made at position 1 than at position 2 on the same side $(p<.01)$, or that both types of responses were made equally on the opposite side by HI Ss. A more reasonable interpretation would be to conclude that exploratory behavior is reflected in $\mathrm{HS}$ and some WhB responses in HI Ss. Some of the WhB responses to the opposite side might also reflect anticipatory behavior as well for HI Ss who had failed to find reinforcement in the $\mathrm{N}$-alley on the goal side.

Our ultimate aims in studying exploratory behavior are to establish the information value that such behavior yields an organism about his environment. Any differential information value of exploratory vs anticipatory behavior will be an important factor to consider in the relationships between motivation level and learning.

\section{REFERENCES}

ADLERSTEIN, A., \& FEHRER, E. The effect of food deprivation on exploratory behavior in a complex maze. J. comp. physiol. Psychol., 1955, $48,250-253$.

COHEN J. S. \& STETTNER, L. J.-Effect of deprivation level on exploratory behavior in the albino rat. J. comp. physiol. Psychol., in press.

DEAUX, E. B., \& PATTEN, R. L. Measurement of the anticipatory goal response in instrumental runway conditioning. Psychon. Sci, 1964, 1, $357-358$.

HUGHES, R. N. Food deprivation and locomotor exploration in the white rat. Anim Behav., 1965, 13, 30-32.

HULL, C. L. Principles of behavior. New York: Appleton-Century-Crofts, 1943.

GLICKMAN, S. E. \& JENSEN, G. D. The effects of hunger and thirst on Y-maze exploration. J. comp. physiol. Psychol., 1961, 54, 83-85.

STACKHOUSE, S. P., BURNS, N. M., \& WOHLFORD, J. Note on exploratory behavior. Psychol. Rep., 1960, 6, 455-457.

\section{NOTE}

1. This study was carried out while J. S. C. was supported by funds from an Experimental Psychology Training Grant to Wayne State University from the National Institute of Mental Health. The authors wish to thank J. Ager and S. S. Komorita for their assistance and advice. 